\title{
ASPECTOS DE LA BIOLOGÍA REPRODUCTIVA DE STEVIA REBAUDIANA (ASTERACEAE)
}

\author{
IRENE CAPONIO ${ }^{1}$, SILVANA C. TORRES ${ }^{2}$, GUILLERMO ANDERSSON ${ }^{3}$ \& GUILLERMO A. NORRMANN ${ }^{1}$
}

\begin{abstract}
Summary: Caponio, I., S. C. Torres, G. Andersson \& G. A. Norrmann. 2016. Aspects of reproductive biology of Stevia rebaudiana (Asteraceae). Bonplandia 25(1): 59-71.

The genus Stevia Cav. (Asteraceae) consists of about 200 species in the Americas. Stevia rebaudiana (Bertoni) Bertoni, originally from Paraguay, is the only cultivated species. It has widely been studied chemically, because of the active compounds found in the leaves. However, there is controversy about its reproductive system, as some authors consider it an obligate apomict, while others a sexually reproducing species. In order to clarify this issue: the megasporogenesis, megagametogenesis, floral phenology, pollen viability, and stigmatic receptivity were studied in three ecotypes, under self-pollination and cross-pollination. According to our results, the plants reproduce sexually and have a self-incompatibility system that prevents self-fertilization.
\end{abstract}

Key words: Floral phenology, megagametogenesis, megasporogenesis, self-incompatibility, stigmatic receptivity.

Resumen: Caponio, I., S. C. Torres, G. Andersson \& G. A. Norrmann. 2016. Aspectos de la biología reproductiva de Stevia rebaudiana (Asteraceae). Bonplandia 25(1): 59-71.

El género Stevia Cav. (Asteraceae) presenta alrededor de 200 especies en el continente americano. Stevia rebaudiana (Bertoni) Bertoni, originaria de Paraguay, es la única especie cultivada. Ha sido ampliamente estudiada desde el punto de vista químico, por los principios activos que posee sus hojas. Sin embargo existe controversia respecto a su sistema reproductivo, ya que es considerada apomíctica obligada por algunos autores, mientras otros la consideran de reproducción sexual. A los efectos de clarificar esta cuestión, se realizaron estudios de: megasporogénesis, megagametogénesis, fenología de la floración, viabilidad de polen y receptividad estigmática en tres ecotipos en condiciones de autopolinización y polinización cruzada. De acuerdo a los resultados obtenidos, las plantas analizadas se reproducen sexualmente y poseen un sistema de autoincompatibilidad que impide la autofertilización.

Palabras clave: Autoincompatibilidad, fenología floral, megagametogénesis, megasporogénesis, receptividad estigmática.

${ }^{1}$ Facultad de Ciencias Agrarias, Universidad Nacional del Nordeste. Sargento Cabral 2131. 3400 Corrientes. E-mail: caponio@agr.unne.edu.ar

${ }^{2}$ Ex alumna Facultad de Ciencias Agrarias, Universidad Nacional del Nordeste.

${ }^{3}$ Empresa Unigenes, Misiones, Argentina. 


\section{Introducción}

El género Stevia Cav. (Asteraceae, Asteroideae, Eupatorieae) cuenta con aproximadamente 200 especies en América (Cabrera, 1978), distribuidas principalmente en regiones tropicales y subtropicales, desde California hasta Argentina.

Stevia rebaudiana (Bertoni) Bertoni, conocida vulgarmente como " $\mathrm{Ka}$ ' $a \mathrm{He}^{\prime} e$ " o "yerba dulce", crece naturalmente sólo en el NE de Paraguay, especialmente en la Sierra de Amambay, y en el sur de Brasil en el estado de Mato Grosso do Sul (Cabrera et al., 1996); habita en diferentes tipos de suelos, prefiriendo los levemente ácidos, arcillo-arenosos, húmedos (Yadav et al., 2010).

De 110 especies del género estudiadas desde el punto de vista organoléptico, sólo $S$. rebaudiana y $S$. phlebophylla A. Gray presentan principios edulcorantes como esteviósidos y rebaudiósidos (glicósidos diterpenos) (Soejarto et al., 1982).

Desde el punto de vista citogenético, el género ha sido ampliamente estudiado; se realizaron recuentos cromosómicos y establecieron las fórmulas cariotípicas en aproximadamente 55 especies de Norte y Centro América (Grashoff et al., 1972; Watanabe et al., 2001). En las especies que habitan en América del Norte, además de diploides, es frecuente encontrar citotipos poliploides: S. elatior Kunth, S. serrata Cav. (King et al., 1976), S. eupatoria (Spreng.) Willd. (Keil \& Stuessy, 1975), S. ovata Willd. (Powell \& Powell, 1978), S. latifolia Benth. (Ralston et al., 1989), S. viscida Kunth, S. pubescens Lag., y S. stenophylla A. Gray (Keil \& Stuessy, 1975; Turner \& Flyr, 1966).

Stevia rebaudiana y las especies sudamericanas estudiadas hasta el momento son diploides con $2 \mathrm{n}=2 \mathrm{x}=22$ cromosomas ( $\mathrm{Li}$ et al., 1982; Frederico et al., 1996; Oliveira et al., 2004; Galiano, 1987; Wulff et al., 1996).

La apomixis es frecuente en las Asteraceae, Rosaceae y Poaceae y en la mayoría de los casos sólo se presenta en especies o citotipos poliploides (Noyes, 2007). Según Nogler (1984), esta distribución podría ser debida a letalidad genética que impide el mantenimiento de la apomixis a nivel diploide. Sin embargo, se conocen diploides apomícticos en
Boechera (Brassicaceae) (Thompson, 2006) y en Paspalum (Poaceae) (Siena et al., 2008; Delgado et al., 2014).

Una revisión crítica de evidencias de apomixis en Asteraceae confirma que de 68 géneros considerados previamente apomícticos, sólo en 22 de ellos está fehacientemente comprobada y se presenta en citotipos ploiploides; en los 46 géneros restantes, las evidencias son erróneas, incompletas o requieren confirmación del modo de formación del gametófito femenino para establecer con certeza el modo de apomixis en los diferentes casos (Noyes, 2007). En varias especies que presentan complejos poliploides, tales como Crepis occidentalis (Gustafsson, 1947), Eupatorium pauciflorum (BertassoBorges \& Coleman, 1998); y en géneros como Hieracium (Fehrer, 2007), Taraxacum (Richards, 1973; van Baarlen, 2002; Wittzell, 1999), Arnica (Barker, 1966), Erigeron (Noyes, 2000) y Antennaria (Nygren, 1954; Bergman, 1951) la apomixis es el sistema de reproducción más frecuente.

En dos especies del género Stevia, King y Robinson (1967) observaron dos patrones de morfología de polen en especies de Norte América: (i) granos de polen de tamaño variable, diferencias en la simetría y forma en los citotipos poliploides; y (ii) los diploides, con un patrón uniforme en tamaño y morfología. Basándose en estas observaciones, Grashoff et al. (1972), afirman que "en la mayoría de las especies herbáceas del género Stevia, el sistema de reproducción que prevalece es la agamospermia y el modo de agamospermia es diplosporia obligada". De acuerdo a este concepto Monteiro (1980) y posteriormente Molero (1984) y Oliveira et al. (2004) sostienen que $S$. rebaudiana se reproduce a través de agamospermia, presentando como evidencias una baja formación de aquenios e inviabilidad del polen en citotipos diploides y poliploides inducidos.

El objetivo principal de este trabajo fue estudiar algunos aspectos de la biología reproductiva de tres ecotipos de S. rebaudiana, analizando la fenología de floración, receptividad estigmática, viabilidad de los granos de polen y los procesos de megasporogénesis y megagametogénesis. 


\section{Material y Métodos}

Las plantas utilizadas corresponden a las localidades de Misiones, Leandro N. Alem (IG) y Gobernador Roca (GR) y de Colón, provincia de Entre Ríos (ER), Argentina. Todas son cultivadas localmente. De cada ecotipo se utilizaron dos plantas, los ejemplares de herbario, fueron depositados en el herbario CTES con la siguiente numeración IC 161, IC 162, IC 163 (IC= Irene Caponio).

Las plantas fueron mantenidas en macetas de 3 litros con sustrato especial preparado en el invernáculo de la Cátedra de Genética y Fitotecnia, de la Facultad de Ciencias Agrarias, UNNE, Corrientes, Argentina.

\section{Fenología reproductiva y receptividad estigmática}

La fenología floral de la especie se interpretó en base a observaciones en flores desde el momento de antesis de la primera flor en el capítulo hasta la marchitez de las flores.

Para conocer la receptividad estigmática se utilizó la técnica de Osborn et al. (1988) que se basa en la actividad de peroxidasas. La respuesta es considerada positiva cuando se produce burbujeo sobre los estigmas al contacto con peróxido de hidrógeno. Esta prueba se realizó en cinco flores de tres capítulos de cada ecotipo, en cada una de las fases establecidas. Se estableció arbitrariamente una gradación de signos $(+)$, según la intensidad de las burbujas observadas.

\section{Adherencia del polen a la superficie estigmática y germinación de granos de polen "in situ"}

La adherencia de los granos de polen a la superficie estigmática y la germinación de granos de polen "in situ" se determinaron por fluorescencia utilizando el método de Kho y Boër (1968), y para ello se utilizaron 87 estigmas tratados con polinizaciones manuales entre flores de los distintos ecotipos (Tabla 1) y 40 con polen de flores de la misma planta, geitonogamia (Tabla 2). Las flores polinizadas se mantuvieron marcadas y aisladas hasta el momento de fijación en FAA.

La fijación del material se realizó después de 8 y $15 \mathrm{~h}$ de realizadas las polinizaciones por geitonogamia y 6,8 y $15 \mathrm{~h}$ de realizadas las polinizaciones en los cruzamientos entre los diferentes ecotipos.

Las observaciones se realizaron en un microscopio de epifluorescencia marca Leica DM2500.

\section{Viabilidad de los granos de polen}

La viabilidad de los granos de polen se estimó utilizando los métodos indirectos de coloración con carmín glicerina (Marks, 1954) $\mathrm{y}$ con lugol (yodo-ioduro de potasio). De cada planta se tomaron estilos de 5 flores, de diferentes capítulos al momento de la antesis los que se colocaron sobre una gota de colorante, moviendo los estilos en el líquido para poder dispersar los granos de polen; se analizaron y contabilizaron alrededor 1000 granos de polen de cada ecotipo.

\section{Megasporogénesis y megagametogénesis}

Para el estudio de la megasporogénesis y megagametogénesis, se fijaron capítulos en diferentes estadios de desarrollo en FAA (etanol $70 \%$ : ácido acético: formaldehído, 9: 0,5: 0,5) durante $24 \mathrm{~h}$ y se conservaron en alcohol $70 \%$ a $4^{\circ} \mathrm{C}$ hasta el momento del disecado. Se utilizaron técnicas histológicas clásicas (Johansen, 1940) seccionando el material a $12 \mu \mathrm{m}$ de espesor y coloreando con safranina-fast green.

\section{Resultados}

Fenología de la fase de floración y receptividad estigmática

Una inflorescencia madura de $S$. rebaudiana posee alrededor de 190 capítulos y aproximadamente el $10 \%$ de los mismos se abren simultáneamente. Cada capítulo de Stevia posee 5 flores, con diferentes estadios de desarrollo.

En $S$. rebaudiana, el androceo está compuesto por cinco anteras soldadas entre sí por sus bordes (sinantéreo) formando un tubo alrededor del estilo; las anteras poseen dos tecas de dehiscencia introrsa longitudinal. El estilo posee en el tercio superior y en la superficie externa pelos colectores, los que arrastran el polen, quedando éste adherido y expuesto (Fig. 1 A-B). La superficie estigmática presenta así 
Tabla 1. Detalle de polinizaciones realizadas entre los diferentes ecotipos.

\begin{tabular}{|cccc|}
\hline $\begin{array}{c}\text { Cruzamientos } \\
\text { realizados }\end{array}$ & $\begin{array}{c}\text { No De Flores } \\
\text { Polinizadas }\end{array}$ & $\begin{array}{c}\text { Horas transcurridas } \\
\text { desde la Antesis a la } \\
\text { Polinización }\end{array}$ & $\begin{array}{c}\text { Horas transcurridas desde la } \\
\text { Polinización a la fijación del material }\end{array}$ \\
\hline IG $\times$ ER & 87 & $2-120$ & 6,8 y 15 \\
\hline ER X IG & 87 & $2-120$ & 6,8 y15 \\
GR $\times$ IG & 87 & $2-120$ & 6,8 y15 \\
Total & 261 & & \\
\hline
\end{tabular}

dos regiones una apical en el tercio superior con pelos largos colectores y en los dos tercios inferiores una zona de receptividad donde en corte transversal se observan los pelos receptivos hacia los bordes laterales de las ramas estigmáticas (Fig. $1 \mathrm{C}$-E).

La apertura de las flores del capítulo no es simultánea (Fig. 2 A) y como sucede en la mayoría de las compuestas, ocurre secuencialmente en dos o tres días.

La antesis comienza cuando el estilo emerge entre las anteras y los pétalos se encuentran expandidos, ocurriendo este proceso durante la mañana entre las 9:30 y 11:00 h dependiendo de la longitud del día (Fig. 2 B). El polen permanece sobre la superficie externa del estilo por un período aproximado de 2-4 h (Fig. 2 C-D).

Con el transcurso de las horas, el estilo continúa elongándose y las ramas estigmáticas comienzan a separarse (Fig. 2 E-G) hasta quedar en ángulo de $180^{\circ}$ a las 24 h. Posteriormente las ramas estigmáticas continúan curvándose (Fig. 2 H-I) quedando deflexos a las 96 h y hasta la marchitez total de la flor. Se observaron que flores no polinizadas (aisladas) mantienen sus estilos y ramas estigmáticas sin marchitarse hasta más de 7 días después de haber emergido; mientras los estigmas polinizados exitosamente, a las $24 \mathrm{~h}$ comienzan a marchitarse.

Adherencia de polen a la superficie estigmática

En las polinizaciones realizadas, se observó que los granos de polen quedan adheridos a los pelos receptores de la parte media e inferior de las ramas estigmáticas (Fig. 1 D-E).

A partir de $6 \mathrm{~h}$ de producida la antesis, cuando las dos ramas estigmáticas están separadas, las mismas reaccionan positivamente a la presencia del peróxido de hidrógeno con burbujeo muy leve $(+)$, en la región de pelos receptores de las ramas estigmáticas, aumentando éste levemente $(++)$ a las $8 \mathrm{~h}$ de ocurrida la antesis. El máximo burbujeo se observó a partir de las $24 \mathrm{~h}$ de ocurrida las antesis, manteniéndose a este nivel $(++++)$ hasta las $72 \mathrm{~h}$ después de la antesis.

Este patrón de receptividad es similar en los diferentes ecotipos; observándose que el máximo valor de receptividad en los ecotipos IG y ER se prolonga por 24 h más (Fig. 3).

La receptividad de los estigmas comienza a partir de $6 \mathrm{~h}$ de producida la antesis, y aumenta

Tabla 2. Autopolinizaciones por geitonogamia realizadas en los distintos ecotipos.

\begin{tabular}{|cccc|}
\hline Autopolinizaciones & $\begin{array}{c}\text { No de Flores } \\
\text { Polinizadas }\end{array}$ & $\begin{array}{c}\text { Horas transcurridas desde la } \\
\text { Antesis a la Polinización }\end{array}$ & $\begin{array}{c}\text { Horas Transcurridas } \\
\text { desde la } \\
\text { Polinización a la fijación }\end{array}$ \\
\hline IG X IG & 40 & $4-8-24 y 48$ & 8 y 15 \\
\hline ER X ER & 40 & $4-8-24$ y 48 & \\
TOTAL & 40 & $4-8-24 y 48$ & \\
\hline
\end{tabular}



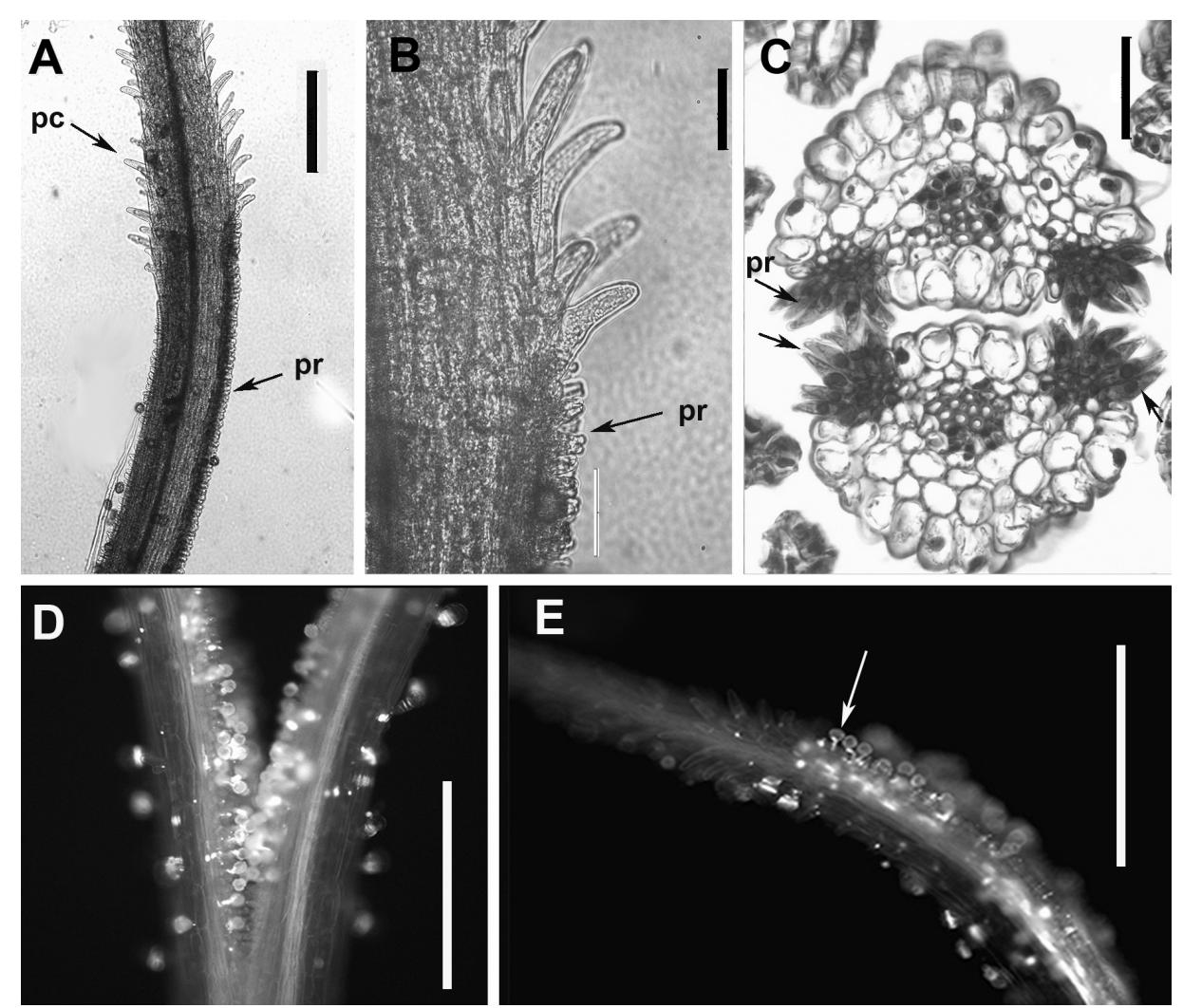

Fig. 1. Áreas de la superficie estigmática receptiva. A: Rama estigmática mostrando superficie con diferentes tipos de pelos; pelos colectores (pc) en el tercio apical y pelos receptivos (pr) en zonas de adherencia del polen o receptiva. B: Detalle de pelos colectores y pelos receptivos (pr). C: Corte transversal de estigma a la altura de zona de receptividad, (pr) pelos receptivos. D-E: Granos de polen adheridos en los $2 / 3$ inferiores de las ramas estigmáticas. Escalas en $\mu \mathrm{m}$ : A: 100; B-C: 20; D-E: 200.

paulatinamente hasta llegar al máximo valor a las $24 \mathrm{~h}$, permaneciendo receptivos hasta 96-144 h después de la antesis, disminuyendo considerablemente la reacción ante el peróxido de hidrógeno. A partir de este momento, por lo general la corola comienza a marchitarse.

Después de las $24 \mathrm{~h}$ de realizada la polinización, las ramas estigmáticas de las flores polinizadas toman una coloración más oscura y se marchitan. Por el contrario, en aquellas flores que no se polinizaron, los estigmas permanecen bien hidratados y poco receptivos, aún cuando las corolas estén marchitas.

\section{Germinación de granos de polen "in situ"}

En flores polinizadas manualmente por geitonogamia (polen de diferentes flores de la misma planta) a partir de las $4 \mathrm{~h}$ y hasta 120 h después de la antesis, se observaron muy pocos granos de polen adheridos (Fig. 4 A-B); de aquellos granos que quedan adheridos el $83 \%$ desarrollaron el tubo polínico, pero en éstos después de $15 \mathrm{~h}$ la longitud del tubo polínico apenas sobrepasaba brevemente el diámetro del grano de polen, deteniéndose el crecimiento sobre los pelos receptores de las ramas estigmáticas (Fig. $4 \mathrm{C}$-E).

En polinizaciones cruzadas se observó mayor adherencia de los granos de polen en las superficies laterales de las ramas estigmáticas (Fig. 4 F-G). A partir de $8 \mathrm{~h}$ de polinizados el $88 \%$ de los granos de polen que estaban adheridos desarrollaron tubo polínico, con una longitud igual o mayor al diámetro del grano de polen. A $10 \mathrm{~h}$ de realizada la polinización, la longitud de los tubos polínicos superaban 1,5 


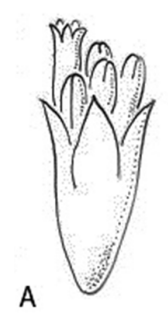

B

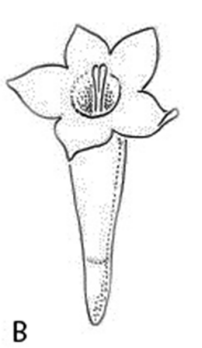

C
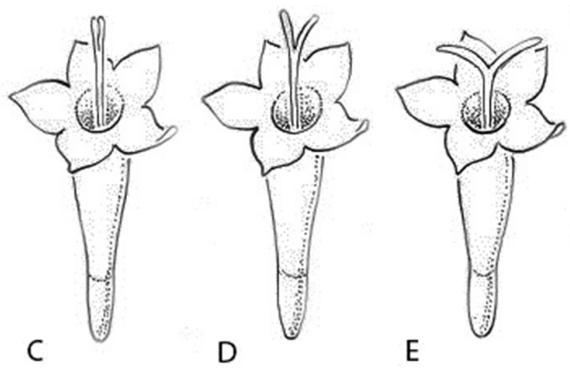

E
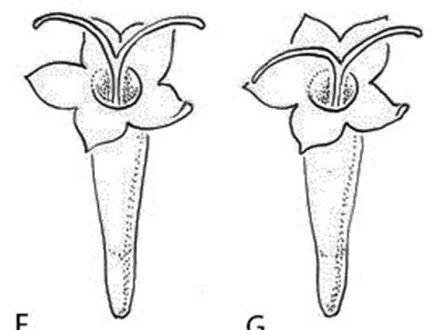

G
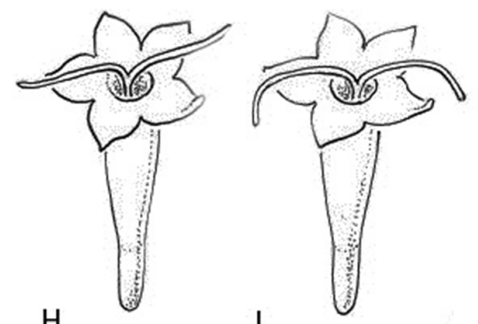

I

Fig. 2. Fenología de floración y receptividad estigmática. A: Apertura de las flores del capítulo en forma secuenciada. B: Longitud de los estigmas en el momento de antesis. C-D: Estigmas a las 2 h de producida la antesis. E-G: Posición de las ramas estigmáticas a las $5 \mathrm{~h}$ de la antesis. H-I: Ramas estigmáticas con mayor receptividad a partir de las $24 \mathrm{~h}$ de la antesis.

veces el diámetro de los granos de polen y éstos atravesaban los pelos receptores (Fig. $4 \mathrm{H}-\mathrm{I}$ ); a las $15 \mathrm{~h}$ el desarrollo de los mismos llegaba hasta el tejido de transmisión y $24 \mathrm{~h}$ después de las polinizaciones los tubos polínicos alcanzaban al tercio inferior del ovario (Fig. 5 A-B). El promedio de granos de polen que desarrollaron tubos polínicos de igual longitud que el diámetro del grano tanto en geitonogamia como en polinizaciones cruzadas con esta técnica fue del $85 \%$. Estos datos sustentan también la viabilidad de los mismos.

En condiciones de autopolinización la detención del crecimiento de los tubos polínicos después de $8 \mathrm{~h}$ sobre la superficie de los pelos receptores confirmaría la presencia de un sistema génico de autoincompatibilidad gametofítica a nivel de estigma, que impide el desarrollo de granos de polen de la misma planta.

\section{Viabilidad del polen}

Los granos de polen en $S$. rebaudiana son 3 -colporados, isopolares y radiosimétricos; esferoidales, pequeños, de 15-20 $\mu$ m de diámetro. Poseen una exina microequinada. Los granos se mantienen unidos formando másulas adhesivas al momento de la antesis.

Por métodos colorimétricos se pueden identificar los granos polen no viables (no coloreados) debido a defectos y/o insuficiencias de citoplasma o de sustancias de reservas. Se comprobó que la coloración de los granos de polen en $S$. rebaudiana, está directamente relacionada al tiempo de exposición en el colorante. Con carmín glicerina, se observó que con 4-8 h de exposición al colorante el citoplasma es levemente rosado (Fig. 6 A-B) y solo después de $48 \mathrm{~h}$ de exposición al colorante el citoplasma colorea intensamente (Fig. 6 C) y se logran diferenciar los granos viables de los estériles. Con esta técnica los porcentajes de granos de polen viables en los diferentes ecotipos fue de 94,8 a 97,9\% (Tabla 3).

Cuando se utiliza lugol, a las $4 \mathrm{~h}$ se observa que todos los granos de polen toman una coloración intensa (Fig. 6 D); después de 8 h de contacto con el colorante comienzan a aclararse (Fig. $6 \mathrm{E}$ ) y a las $48 \mathrm{~h}$ se diferencian fácilmente los granos de polen coloreados (viables) tomando una coloración amarillenta (debido a la presencia 


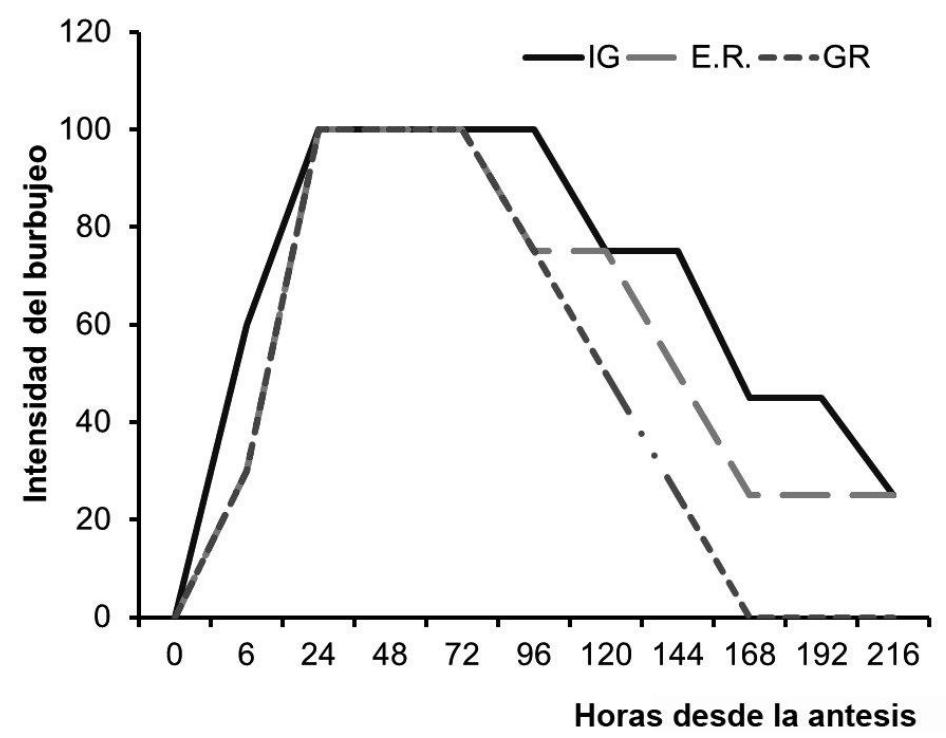

Fig. 3. Patrón de receptividad de los diferentes cultivares, por el método de Osborn et al. (1988). Los estigmas permanecen receptivos hasta más de 196 h después de haber emergido.

de inulina como sustancia de reserva) de los no coloreados, considerados no viables (Fig. 6 F). Los valores obtenidos con esta técnica variaron entre $95,3 \%$ y $96,4 \%$ en los diferentes ecotipos.

Con el objeto de establecer si existían diferencias entre ambas técnicas de coloración se aplicó la prueba $\chi^{2}$, comprobando que solo existían diferencias significativas entre ecotipo y no entre los colorantes utilizados.

\section{Megasporogénesis y megagametogénesis}

En $S$. rebaudiana el ovario es unilocular y uniovulado (Fig. 7 A). El óvulo a la madurez es anátropo, unitégmico y tenuinucelado. Durante estadios tempranos del desarrollo, células de la epidermis interna del integumento se elongan radialmente y adquieren un citoplasma denso; estas células conforman el endotelio. Cuando se diferencia la célula madre de la megáspora (CMM), el tejido nucelar está reducido a una sola capa de células; la CMM es notable con un núcleo conspicuo y nucléolo prominente (Fig. 7 B). Ésta se divide por meiosis, originando una tétrada lineal de megásporas. Posteriormente, la megáspora ubicada hacia el extremo chalazal desarrollará el megagametófito mientras que las megásporas restantes situadas en el extremo micropilar

Tabla 3. Viabilidad de polen por métodos colorimétricos indirectos. Se consigna el porcentaje de granos de polen viable, cantidad de granos de polen coloreados/no coloreados contados.

\begin{tabular}{|c|c|c|c|c|}
\hline \multirow{2}{*}{ Tratamiento } & \multicolumn{4}{|c|}{ Ecotipo } \\
\hline & IG & ER & GR & Total / Tratamiento \\
\hline \multirow{2}{*}{ Carmín - glicerina } & $97,46 \%$ & $94,82 \%$ & $97,96 \%$ & \multirow{2}{*}{4177} \\
\hline & 2379 / 62 & $1209 / 66$ & $589 / 12$ & \\
\hline \multirow{2}{*}{ Lugol } & $95,35 \%$ & $95,35 \%$ & $96,43 \%$ & \multirow{2}{*}{3099} \\
\hline & $1218-(49)$ & $1150-(56)$ & $731 / 27$ & \\
\hline Total / Accesión & 3708 & 2481 & 1359 & \\
\hline
\end{tabular}



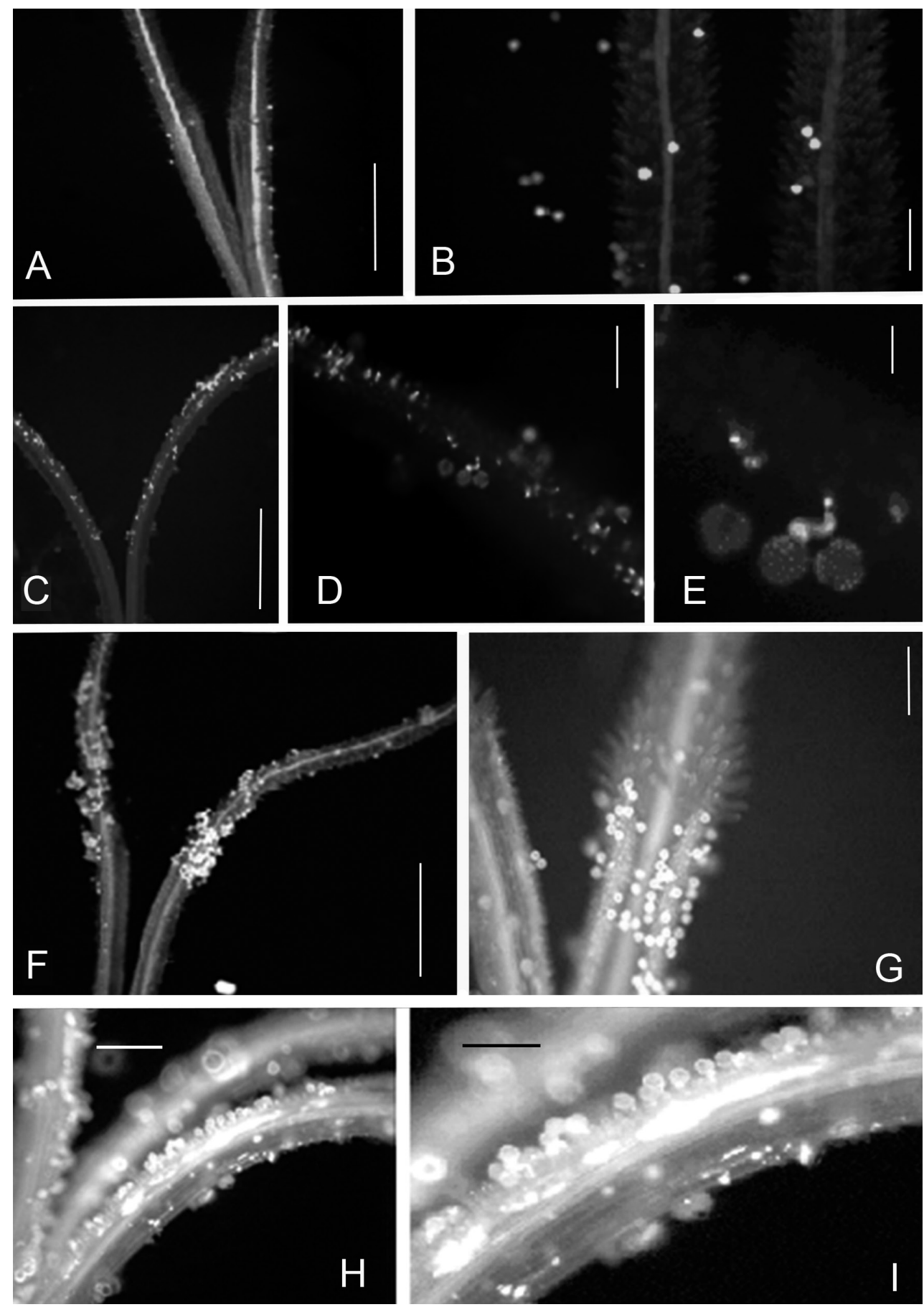

Fig. 4. Adherencia y germinación de granos de polen en condiciones de autopolinización por geitonogamia y polinización cruzada. A-B: Estigma autopolinizado a las $4 \mathrm{~h}$ de la emergencia de los estigmas. C-D: Estigmas autopolinizados a $6 \mathrm{~h}$ de producida la antesis se observa adherencia de los granos de polen en la región de pelos receptores. E: Estigmas autopolinizados a las $8 \mathrm{~h}$ de producida la antesis y fijados después de $15 \mathrm{~h}$ de la polinización, el tubo polínico no sobrepasa el diámetro del grano de polen. F-G: Estigmas con polen de otros cultivares $6 \mathrm{~h}$ después de polinizados. H-I: Estigmas polinizados con polen de cultivares diferentes, después de $15 \mathrm{~h}$ de polinizados, detalle del desarrollo de los tubos polínicos en los estigmas. Escalas en $\mu$ m: A, C, E, H, I: 200; B, D, F: 100; G: 20. 
degeneran (Fig. 7 C). Luego la megáspora funcional se alarga y su núcleo se divide originando un saco binucleado con sus núcleos ubicados uno en cada polo (Fig. 7 D). Ya en los polos, cada núcleo experimenta dos divisiones mitóticas consecutivas originándose primero un saco embrionario 4-nucleado pasando luego a 8-nucleado (Fig. 8 A). Posteriormente, un núcleo de cada polo migra a la región central, y comienza el proceso de citocinesis, quedando formado un megagametófito maduro con 7 células y 8-núcleos (Fig. 8 B). Generalmente en las células ubicadas hacia el extremo calazal ocurren una o más divisiones posteriores, quedando formadas normalmente cuatro o más antípodas lineales.

Las células ubicadas hacia el extremo micropilar originan dos células alargadas, las sinérgidas, e inmediatamente por arriba de ellas se diferencia una célula redondeada con citoplasma denso y un gran núcleo con nucléolo prominente, la ovocélula u oósfera. En el centro se ubica la célula media muy vacuolizada, con dos núcleos polares y grandes nucléolos. Los núcleos polares al momento de producirse la fecundación se observan fusionados (Fig. 8 C, D).

De 343 óvulos estudiados de los tres ecotipos, se observó que el promedio de óvulos abortados a lo largo de los diferentes estadios de desarrollo fue de $4 \%$, no observándose diferencias entre los diferentes ecotipos.

\section{Discusión y Conclusiones}

La capacidad de autopolinización espontánea en diferentes especies de Asteraceae, entre ellas especies del género Stevia, ha sido estudiada por Morales \& Galetto (2003) quienes determinaron que en su mayoría requieren la presencia de insectos para desarrollar los frutos.

En este trabajo se demuestra que en $S$. rebaudiana, tanto por el sistema de presentación del polen a polinizadores, como por diferencias temporales en la receptividad estigmática existe protandria. La diferenciación de una región de receptividad menor que la longitud de las ramas estigmáticas y la presencia de un sistema génico de autoincompatibilidad sustentan que esta especie es esencialmente alógama.

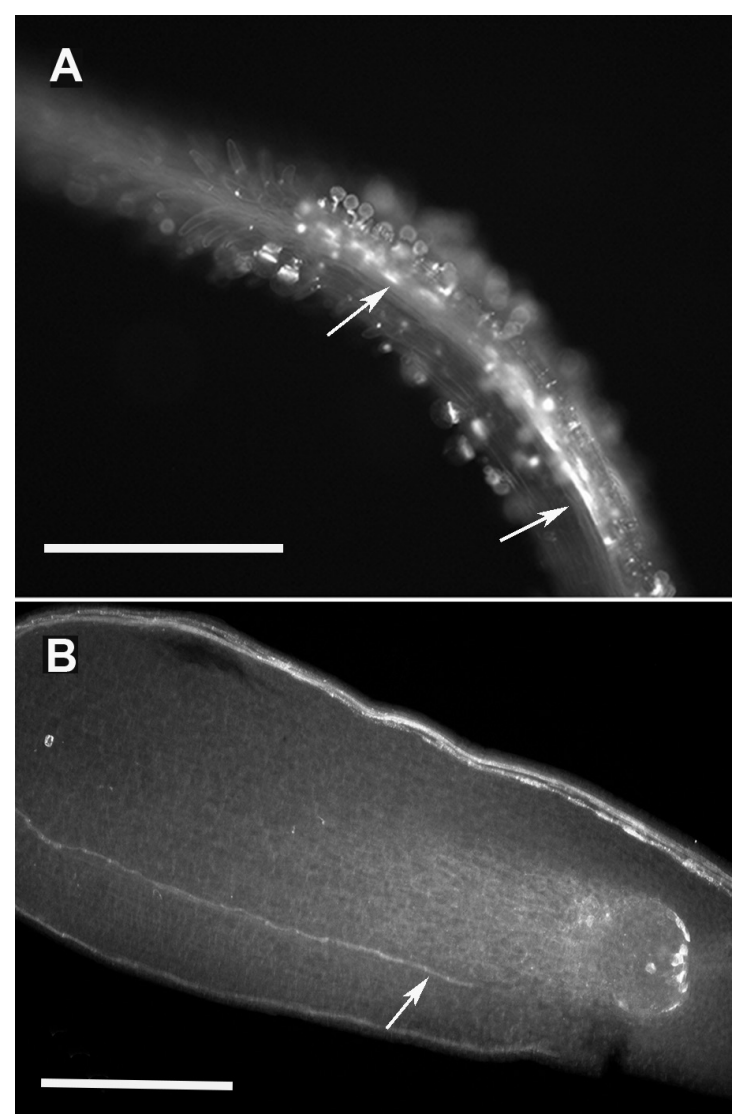

Fig. 5. Germinación de granos de polen "in situ". A: Granos de polen adheridos en las ramas estigmáticas y tubos polínicos bien desarrollados (flechas) después de $8 \mathrm{~h}$ de polinizados. B: Desarrollo del tubo polínico (flecha) después de $24 \mathrm{~h}$ de realizada la polinización entre cultivares diferentes. Escala: A-B: $100 \mu \mathrm{m}$.

Los resultados obtenidos para viabilidad de los granos de polen por coloración de citoplasma y sustancias de reserva, dos técnicas que miden patrones diferentes, indican que solo aproximadamente el 4\% de los granos de polen presentan problemas de viabilidad citoplásmica o bien del contenido de reserva. Estos datos contrastan con los observados por Oliveira et al. (2004) quienes reportan que el 100\% de granos de polen son inviables en citotipos diploides. La formación normal de tétrades con valores superiores al 93\% obtenidos por Oliveira et al. (2004), sugeriría un proceso meiótico normal, sin irregularidades, por lo tanto deberían observar porcentajes mayores de granos de polen viables. 


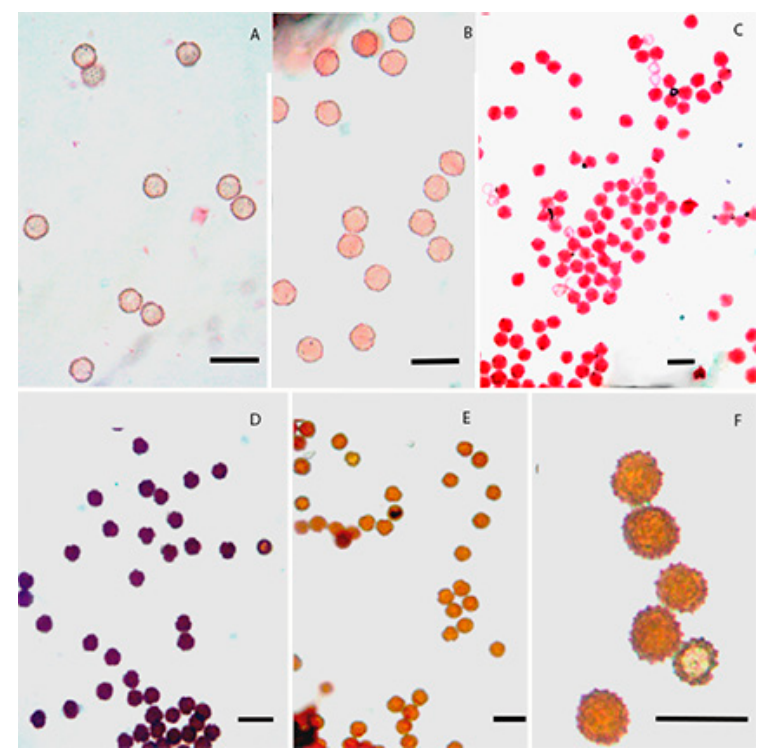

Fig. 6. Viabilidad de polen por métodos colorimétricos. A-C: Carmín-glicerina. D-F: Coloración con Iodo-Ioduro de Potasio (Lugol). A y D: 4 h B y E: 24 h Cy F: 48 h de exposición al colorante. Escala: 50 um.

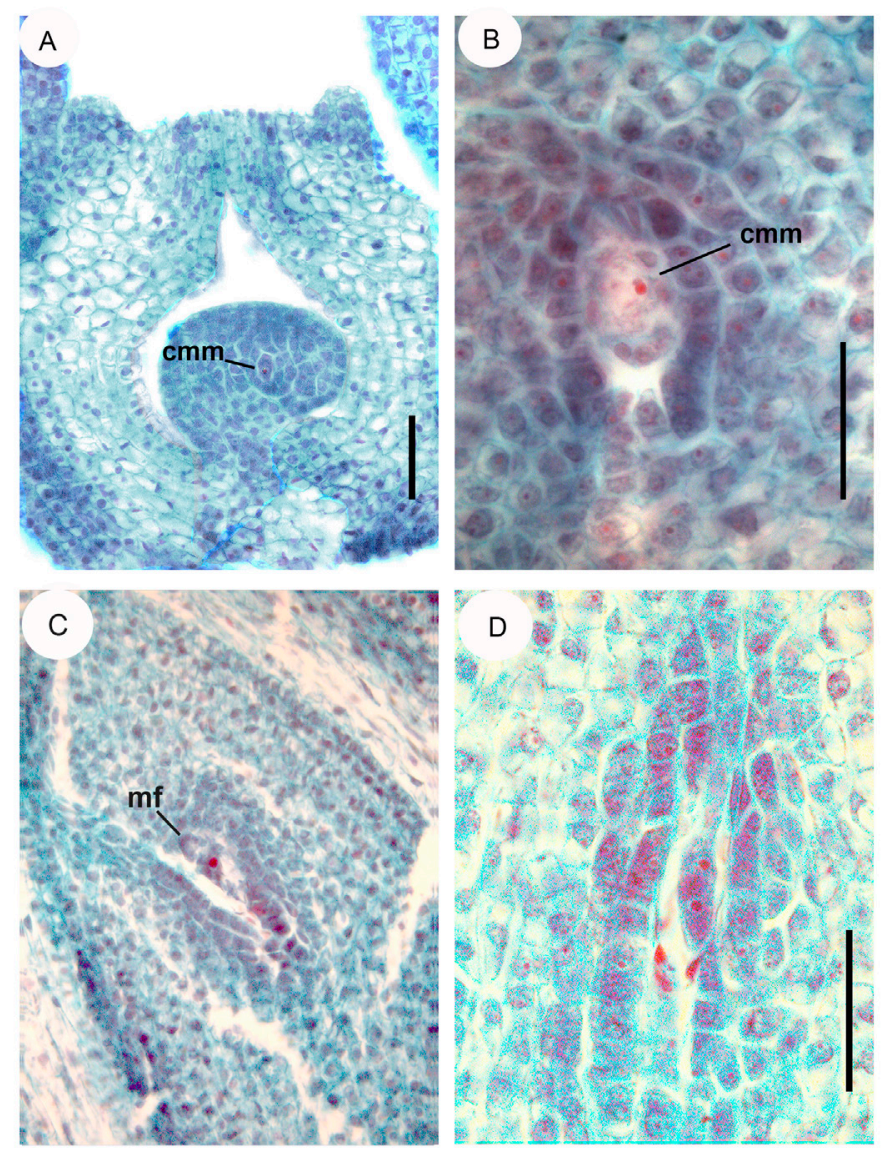

Fig. 7. Megasporogénesis. A: Óvulo joven con CMM diferenciada. B: Detalle de la CMM. C: Megáspora funcional (mf). D: Saco embrionario binucleado. Escalas en $\mu \mathrm{m}$ : A, C-D: 50; B: 20. 


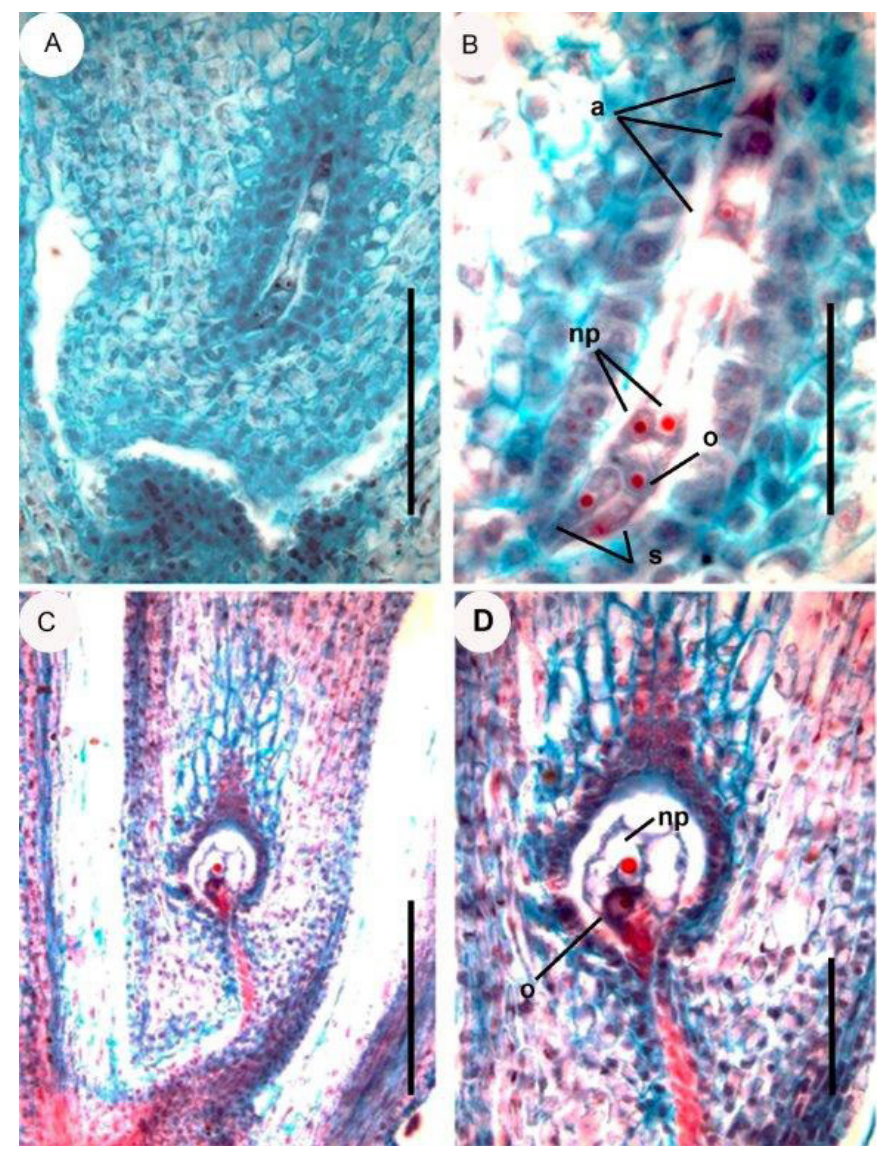

Fig. 8. Megagametogénesis. A: Saco embrionario 8-nucleado. B: Detalle sinérgidas (sg), ovocélula (o), núcleos polares (np) antípodas (a). C: Megagametófito fecundado. D: Detalle del megagametófito fecundado, núcleos polares fusionados (np) oósfera (o). Escalas en $\mu$ m: A y C: 200; B y D: 50.

El porcentaje de granos de polen germinados "in vivo" observados en este trabajo con técnicas de fluorescencia $(85 \%$ de granos germinados) coincide con lo obtenido, o es ligeramente menor al registrado por los métodos indirectos de coloración.

De acuerdo al desarrollo de los sacos embrionarios en $S$. rebaudiana, se pueden tipificar como sacos monospóricos, tipo Polygonum, bipolar, 8-nucleados, característicos de especies que presentan reproducción sexual, coincidiendo con lo observado por Shaffert \& Chebotar (1984). De 183 óvulos estudiados en estadios jóvenes de desarrollo (megasporogénesis y megagametogénesis) ninguno de ellos presentó indicios que pudieran sugerir la existencia de procesos que suceden en los diferentes tipos de apomixis gametofítica (diplosporia o aposporia). No se observaron anomalías en el proceso meiótico, ni formación de núcleos de restitución que pudieran indicar la existencia de "diplosporia obligada por medio de división pseudohomeotípica", como afirman Grashoff (1974) y Oliveira et al. (2004). Estos autores sostienen que $S$. rebaudiana se reproduce a través de agamospermia (apomixis), presentando solo como evidencias el porcentaje de formación de aquenios. Tampoco se observaron embriones prematuros o proembriones, por lo que se descarta la posibilidad de la existencia de agamospermia en $S$. rebaudiana.

En consecuencia, los resultados de nuestra investigación son conclusivos en este aspecto. Todos los procesos de gametogénesis hasta formación de aquenios son normales y garantizan la reproducción sexual en Stevia. 


\section{Agradecimientos}

Este trabajo se realizó a través del PI A 17/ 2010-2013 otorgado por la Secretaría General de Ciencia y Técnica de la UNNE. Se agradece al Instituto de Botánica del Nordeste, a la Lic. Stella Maris Pire por su colaboración en la descripción palinológica, a la ilustradora Lic. Laura Simón por los dibujos y a la Cooperativa Agroindustrial de Misiones (CTM) por ceder material para este estudio.

\section{Bibliografía}

BARKER, W. 1966. Apomixis in the genus Arnica (Compositae). PhD Dissertation, University of Washington, Seattle. 142 pp.

BERGMAN, B. 1951. On the formation of reduced and unreduced gametophytes in the female of Antennaria carpatica. Hereditas 37: 501-518.

BERTASSO-BORGES, M. \&. J. R. COLEMAN. 1998. Embryology and cytogenetics of Eupatorium pauciflorum and E. intermedium (Compositae). Genet. Mol. Biol. 21: 507-514.

CABRERA, A. L. 1978. Flora De La Provincia De Jujuy, República Argentina: Parte X-Compositae. Col. Sci. INTA XIII, Buenos Aires.

CABRERA, A. L., W. C. HOLMES \& S. McDANIEL. 1996. Stevia, Compositae III. En SPICHIGER R. y L. RAMELLA (eds.), Flora del Paraguay 25: 302330. Conservatoire et Jardin Botaniques de la Ville de Genève, Missouri Botanical Garden.

DELGADO L., F. GALDEANO, M. E. SARTOR, C. L. QUARIN, F. ESPINOZA \& J. P. A. ORTIZ. 2014. Analysis of variation for apomictic reproduction in diploid Paspalum rufum. Ann. Bot. 113: 1211-1218.

FREDERICO, A., P. RUAS, M. MARIN-MORALES, C. RUAS, \& J. NAKAJIMA. 1996. Chromosomes studies in some Stevia Cav. (Compositae) species from southern Brazil. Rev. Bras. Genet. 19: 605-609.

FEHRER, J. B. 2007. Incogruent plastid and nuclear DNA phylogenies reveal ancient intergeneric hybridization in Pilosella hawkweeds (Hieracium, Chicorieae,Asteraceae). Molec. Phylogenet. Evol. 42: 347-361.

GALIANO, N. 1987. Estudios cromosómicos en especies Argentinas de Stevia (Compositae). Darwiniana 28 : 311-315.

GRASHOFF, J. L. BRIENNER \& D. NORTHINGTON. 1972. Chromosome number in North and Central american Compositae. Brittonia 24: 379-394.

GRASHOFF, J. L. 1974. Novelties in Stevia (Compositae: Eupatoireae). Brittonia. 26: 347-384.

GUSTAFSSON, A. 1947. Apomixis in higher plants.
Lunds Universitets Arsskrift. 100 pp.

JOHANSEN, D. 1940. Plant Microtechinique. McGrawHill Book Company, Inc. NewYork and London. $511 \mathrm{pp}$.

KEIL, D. \& T. STUESSY. 1975. Chromosome counts of Compositae from the United States, Mexico and Guatemala. Rhodora 7: 171-195.

KHO, Y. O. \& J. BOËR. 1968. Observing pollen tubes by means fluorescence. Euphytica 17: 298-302.

KING, M. \& H. ROBINSON. 1967. Multiple pollen forms in two species of the genus Stevia (Compositae). Sida 3: 165-169.

KING, M., KYHOS, D., POWELL, A., RAVEN, P. \& ROBINSON, H. 1976. Chromosome numbers in Compositae. XIII. Eupatorieae. Ann. Missouri Bot. Gard. 63: 862-888.

LI, L., B. XIU, P. CHANG, \& D. NI 1982. Observations Chromosomes of Stevia rebaudiana from tube seedling. J. Bot. 6: 7.

MARKS, G. 1954. An acetocarmine glycerol jelly for use in pollen fertility counts. Stain Technol. 29: 277.

MOLERO, F. J. 1984. El ka'a he'e, Stevia rebaudiana (Bertoni) Bertoni, Análisis bibliográfico y anotaciones hortícolas. Ministerio de Agricultura y Ganadería. Proyecto de intensificación en fincas pequeñas. USAID-CREDICOOP. Asunción, Par. Publicación Miscelánea $\mathrm{N}^{\circ} 11.75 \mathrm{pp}$.

MONTEIRO, R. 1980. Taxonomia e biologia da reprodução de Stevia rebaudiana Bert. Masters Thesis, Universidade Estadual de Campinas. Campinas, Brasil.

MORALES, C. \&. L. GALETTO. 2003. Influence of compatibility system and life form on plant reproductive success. Plant Biol. 5: 567-573.

NOGLER, G. 1984. Gametophytic apomixis. En JOHRI B. M. (ed.), Embryology of Angiosperms 475-518 pp. Berlin: Springer-Verlag.

NOYES, R. 2000. Diplospory and parthenogenesis in sexual x agamospermous Erigeron compositus (Asteraceae). Amer. J. Bot. 83: 1292-1303.

NOYES, R. 2007. Apomixis in Asteraceae: Diamonds in the rough. Funct. Plant Sci. Biotechnol. 1: 207-222.

NYGREN, A. 1954. Apomixis in angiosperms II. Bot. Rev. 20: 577-649.

OLIVEIRA, V., E. FORNI-MARTINS, P. MAGALHAES \& M. ALVES. 2004. Chromosomal and morphological studies of diploid and polyploid cytotypes of Stevia rebaudiana (Bertoni) Bertoni (Eupatorieae, Asteraceae). Genet. Mol. Biol. 27: 215-222.

OSBORN, M., P. KEVAN \& A. MEREDITH. 1988. Pollination biology of Opuntia polycantha and Opuntia phaecantha (Cactaceae) in Southern Colorado. Plant Syst. Evol. 159 : 139-144.

POWELL, A. \& S. POWELL. 1978. Chromosome numbers in Asteraceae. Madroño 25: 160-169.

RALSTON, B., G. NELSON \& B. TURNER. 1989. 
Documented plant chromosome number I. Chromosome numbers in Mexican Asteraceae with special reference to the Tribe Tagetae. Sida 13: 359-368.

RICHARDS, A. 1973. The origen of Taraxacum agamospecies. Bot. J. Linn. Soc. 66: 189-211.

SIENA L. A., M. E. SARTOR, F. ESPINOZA \& J. P. ORTIZ 2008. Genetic and embryological evidences of apomixis at the diploid level in Paspalum rufum support recurrent auto-polyploidization in the species. Sex. Plant Reprod. 21: 205-215.

SHAFFERT, E. E. \& A. A. CHEBOTAR. 1984. Development of Stevia rebaudiana, Asteraceae female gametophyte under introduction of the Southern Coast. Bul. Acad. de Ştiinţe a Republicii Moldova. Şt. biologice şi chimice: 3-9. (Idioma: Ruso, Abstract Inglés).

SOEJARTO, D., A KINGHORN \& N. FARNSWORTH. 1982. Potential sweeting agents of plant origin III. Organoleptic evaluation of Stevia leaf herbarium samples for sweetness. J. Nat. Prod. 45: 590-599.

THOMPSON, S. K. 2006. A novel mating system analysis for modes of self-oriented mating applied to diplooid and polyploid artic Easter daisies (Townsendia hookeri). Heredity 97: 119-126.

TURNER, B. \& D. FLYR. 1966. Chromosome number in the Compositae. X North American species. Amer. J. Bot. 33: 24-33.

VAN BAARLEN, P. J. 2002. Comparative cytoembryological investigations of sexual and apomicti dandelions (Taraxacum) and their apomictic hybrids. Sex. Plant Reprod. 15: 31-38.

WATANABE, K., T. YAHARA, A. SOEJIMA \& M. ITO. 2001. Mexican species of the genus Stevia (Eupatprieae, Asteraceae). Chromosome numbers and geographical distribution. P1. Spec. Biol. 16: 49-68.

WITTZELL, H. 1999. Chloroplast DNA variation and reticulate evolution inm sexual and apomictic sections of dandelions. Molec. Ecol. 8: 2023-2035.

WULFF A. F., J. H. Hunziker \& A. Escobar. 1996. Estudios Cariológicos en Compositae VII. Darwiniana 34: 213-231.

YADAV, A., S. SINGH, D. DHYANI, \& P. AHUJA. 2010. A review on the improvement of Stevia (Stevia rebaudiana Bertoni). Can. J. Plant. Sci. 91: 1-27.

Original recibido el 9 de marzo de 2016; aceptado el 20 de mayo de 2016. 
